\title{
Circulating tumor cells, tumor-derived extracellular vesicles and plasma cytokeratins in castration-resistant prostate cancer patients
}

\author{
Afroditi Nanou1, Frank A.W. Coumans ${ }^{2}$, Guus van Dalum ${ }^{3}$, Leonie L. Zeune ${ }^{1,4}$, David \\ Dolling ${ }^{6}$, Wendy Onstenk ${ }^{5}$, Mateus Crespo ${ }^{6}$, Mariane Sousa Fontes ${ }^{6,7}$, Pasquale \\ Rescigno $^{6,7}$, Gemma Fowler ${ }^{6}$ Penny Flohr ${ }^{6}$, Christoph Brune ${ }^{4}$, Stefan Sleijfer ${ }^{5}$, \\ Johann S. de Bono ${ }^{6,7}$ and Leon W.M.M. Terstappen ${ }^{1}$ \\ ${ }^{1}$ Department of Medical Cell BioPhysics, MIRA Institute, University of Twente, Enschede, the Netherlands \\ ${ }^{2}$ Department of Biomedical Engineering and Physics, Academic Medical Center, University of Amsterdam, the Netherlands \\ ${ }^{3}$ Department of General, Visceral and Pediatric Surgery, University Hospital and Medical Faculty of the Heinrich-Heine \\ University, Düsseldorf, Germany \\ ${ }^{4}$ Department of Applied Mathematics, MIRA Institute and Faculty of EEMCS, University of Twente, Enschede, the Netherlands \\ ${ }^{5}$ Department of Medical Oncology, Erasmus MC - Cancer Institute, Rotterdam, The Netherlands \\ ${ }^{6}$ Division of Clinical Studies, The Institute of Cancer Research, London, United Kingdom \\ ${ }^{7}$ Prostate Cancer Targeted Therapies Group, The Royal Marsden NHS Foundation Trust, London, United Kingdom \\ Correspondence to: Afroditi Nanou, email: a.nanou@utwente.nl \\ Leon W.M.M. Terstappen, email: I.w.m.m.terstappen@utwente.nl \\ Keywords: circulating tumor cells (CTCS); tumor-derived Extracellular Vesicles (tdEVs); cytokeratin 18 (CK18); caspase-cleaved \\ cytokeratin 18 (CCCK18); castration-resistant prostate cancer (CRPC) \\ Received: November 07, $2017 \quad$ Accepted: March 17, $2018 \quad$ Published: April 10, 2018 \\ Copyright: Nanou et al. This is an open-access article distributed under the terms of the Creative Commons Attribution License \\ 3.0 (CC BY 3.0), which permits unrestricted use, distribution, and reproduction in any medium, provided the original author and \\ source are credited.
}

\section{ABSTRACT}

Purpose: The presence of Circulating Tumor Cells (CTCs) in Castration-Resistant Prostate Cancer (CRPC) patients is associated with poor prognosis. In this study, we evaluated the association of clinical outcome in 129 CRPC patients with CTCs, tumor-derived Extracellular Vesicles (tdEVs) and plasma levels of total (CK18) and caspase-cleaved cytokeratin 18 (ccCK18).

Experimental Design: CTCs and tdEVs were isolated with the CellSearch system and automatically enumerated. Cut-off values dichotomizing patients into favorable and unfavorable groups of overall survival were set on a retrospective data set of 84 patients and validated on a prospective data set of 45 patients. Plasma levels of CK18 and CCCK18 were assessed by ELISAs.

Results: CTCs, tdEVs and both cytokeratin plasma levels were significantly increased in CRPC patients compared to healthy donors (HDs). All biomarkers except for CCCK18 were prognostic showing a decreased median overall survival for the unfavorable groups of 9.2 vs $21.1,8.1$ vs 23.0 and 10.0 vs 21.5 months respectively. In multivariable Cox regression analysis, tdEVs remained significant.

Conclusions: Automated CTC and tdEV enumeration allows fast and reliable scoring eliminating inter- and intra- operator variability. tdEVs provide similar prognostic information to CTC counts. 


\section{INTRODUCTION}

The presence of Circulating Tumor Cells (CTCs) in Castration-Resistant Prostate Cancer (CRPC) as detected by the CellSearch system is associated with poor outcome compared to patients without detectable CTCs [1-6]. Previously, we showed that the presence of small and large tumor microparticles with or without nucleus, positive for Epithelial Cell Adhesion Molecule (EpCAM) and Cytokeratin (CK) and negative for the leukocyte marker CD45 are also associated with poor outcome in CRPC patients [7]. These tumor microparticles can also be measured using the CellSearch system but do not meet the stringent criteria for CTCs. In the present study, we investigate the clinical relevance of both EpCAM+ CK+ CD45- tumor microparticles without a nucleus in blood, defined here as tumor-derived Extracellular Vesicles (tdEVs) and soluble cytokeratins in plasma of CRCP patients. Our interest in cytokeratin plasma levels arises from the fact that one of the prerequisites for a cell and an EV isolated by the CellSearch system in blood to be defined as CTC and tdEV respectively is their cytokeratin expression because of their epithelial origin. Therefore, we additionally investigated whether soluble cytokeratins present in plasma may be associated with clinical outcome and applied as a surrogate biomarker. Measurements of plasma levels of cytokeratin 18 (CK18) and caspase-cleaved cytokeratin 18 (ccCK18) were performed using M65 and M30 ELISAs respectively [8]. CTCs and tdEVs were imaged with the CellSearch system [9] and automatically enumerated with ACCEPT software to avoid interoperator variability [10]. Association of CTCs, tdEVs, CK18 and ccCK18 with clinical outcome in advanced CRPC patients was assessed by Kaplan-Meier plots of Overall Survival (OS), uni-, and multi- variable Cox regression analyses.In this study, two data sets were used: a retrospective data set of 84 patients to determine the cut-off values of CTCs and tdEVs for favorable and unfavorable prognosis and a prospective data set of 45 patients to validate the selected cut-off values. The plasma samples of the two data sets were collected in a different way; hence, different cut-off values for CK18 and ccCK18 were used for each one of them.

\section{RESULTS}

\section{Patient characteristics}

Blood draws of the CRPC patients consisting the retrospective data set (IMMC 38 study) were performed between October 2004 and February 2006. Their average age was 70 years (range $49-87$ years). Blood draws of the CRPC patients consisting the prospective data set were performed between March 2015 and August 2015. Their average age was 68 years (range: $49-83$ years). The values of serum markers (PSA, LDH, ALP,Alb,Hb), age, Gleason score and ECOG performance status for all patients of both data sets are shown in Supplementary Table 1. The site of metastases, prior radiation, surgery and treatment of both data sets are summarized in Supplementary Table 2 . The prospective data set seem to have more advanced disease compared to the retrospective data set, since significantly more patients underwent chemotherapy (91.1\% versus $35.7 \%)$. Furthermore, $82.2 \%$ and $46.7 \%$ of the patients of the prospective data set were already resistant to abiraterone and enzalutamide respectively, indicating more progressed disease compared to the retrospective dataset, where some patients were still undergoing hormone therapy.

\section{Number of CTCs and tdEVs in $7.5 \mathrm{~mL} \mathrm{of} \mathrm{blood}$}

CTCs (Figure 1) and tdEVs (Supplementary Figure 1) were enumerated from the stored digital images using ACCEPT software. We compared manually defined CTCs by a human operator using standard CellSearch criteria for recognition $(\mathrm{CK}+, \mathrm{CD} 45-$, DAPI,$+>4 \mu \mathrm{m})$ versus the automated CTCs defined by a preconfigured quantitative ACCEPT gate for 129 CRPC patients and 16 healthy controls, see Supplementary Figure 2. Linear regression resulted in "automated CTCs" $=0.82$ "manual CTCs" + $0.49\left(\mathrm{R}^{2}=0.882\right)$.

The median, min, max, 25 and 75 percentiles (p25, p75 respectively) and InterQuartile Range (IQR) values of CTCs and tdEVs detected in the 16 healthy controls, the retrospective data set of 84 and the prospective data set of 45 CRPC patients are depicted in Figure 2. The number of CTCs and tdEVs were significantly higher in the CRPC patients as compared to HDs (both $p<0.001$, MannWhitney $U$ test). There were no significant differences for CTCs nor for tdEVs between the two data sets ( $p=0.81$ for CTCs and $p=0.32$ for tdEVs, Mann Whitney $U$ test).

Spearman's rho correlation test showed that CTCs and tdEVs of the full data set of 129 CRPC patients were correlated (Spearman's $\mathrm{R}=0.856, p<0.01$ ). The scatterplot of that data is shown in Supplementary Figure 2.

\section{CK18 and ccCK18 concentrations in plasma samples}

The determined CK18 and ccCK18 concentrations of 16 HDs, 84 retrospective and 45 prospective CRPC patients are illustrated in Figure 2. The concentrations of CK18 and ccCK18 were significantly higher in the CRPC patients compared to the healthy controls $(p<0.001$, Mann-Whitney $U$ test). Unexpectedly, the concentrations of CK18 and ccCK18 were also significantly higher in retrospective data set as compared to the prospective one ( $p<0.001$, Mann-Whitney $U$ test). Further investigation (Supplementary Figure 4) revealed that the main contributor was the use of EDTA plasma in IMMC38 study versus the CellSave plasma samples in the prospective study. 
Given the different values obtained in the two data sets, correlations between CTCs, tdEVs, CK18, ccCK18 and the serum markers of the patients were evaluated within each data set and not in the full data set, Supplementary Table 3. Using Spearman's Rho test, CTCs and tdEVs were strongly correlated.

\section{Determination of cut-off values for CTCs, tdEVs, CK18 and ccCK18}

ROC curves were generated on the retrospective data set to determine the cut-off values for CTCs, tdEVs, CK18, and ccCK18 dichotomization into favorable and unfavorable patient groups for graphical representation. We set dichotomization cut-off values for CTCs, tdEVs, CK18, and ccCK18 on the value that led to equal sensitivity and specificity (value of biomarker for which absolute (sensitivity-specificity) was minimum), Supplementary Figure 3. The cut-off values were 5 for CTCs, 105 for tdEVs, $576 \mathrm{U} / \mathrm{L}$ for CK18 and $265 \mathrm{U} / \mathrm{L}$ for ccCK18. tdEVs performed the best in terms of sensitivity and specificity to predict OS of CRPC patients (having the largest AUC and the highest significance), followed by CTCs and CK18.
Because of the large differences of CK18 and ccCK18 concentrations between the two data sets, we also determined dichotomization cut-off values on the prospective data set, these were $232 \mathrm{U} / \mathrm{L}$ for CK18 (was $576 \mathrm{U} / \mathrm{L}$ ) and $81 \mathrm{U} / \mathrm{L}$ for ccCK18 (was $265 \mathrm{U} / \mathrm{L}$ ).

\section{CTCs, tdEVs, CK18, and ccCK18 versus overall survival of CRPC patients}

Kaplan-Meier plots for patients with favorable and unfavorable CTC and tdEV counts are shown in Figure 3. Median OS of patients in the unfavorable CTC group was 9.2 months, versus 21.1 months in the favorable CTC group $(p<0.001)$. Similarly for tdEVs, median OS of patients in the unfavorable tdEV group was 8.1 versus 23.0 months in the favorable tdEV group $(p<0.001)$.

Kaplan-Meier plots for CK18 and ccCK18 are shown in Figure 4. The retrospective data set is shown in panels A/D. The prospective data set is shown in panels $\mathrm{B} / \mathrm{E}$, and the full data set is shown in panels $\mathrm{C} / \mathrm{F}$. The two data sets were dichotomized using different cut-off values, because the plasma samples were collected in a different way. However, in both data sets CK18 was prognostic of OS with the HRs as well as the relative number of patients
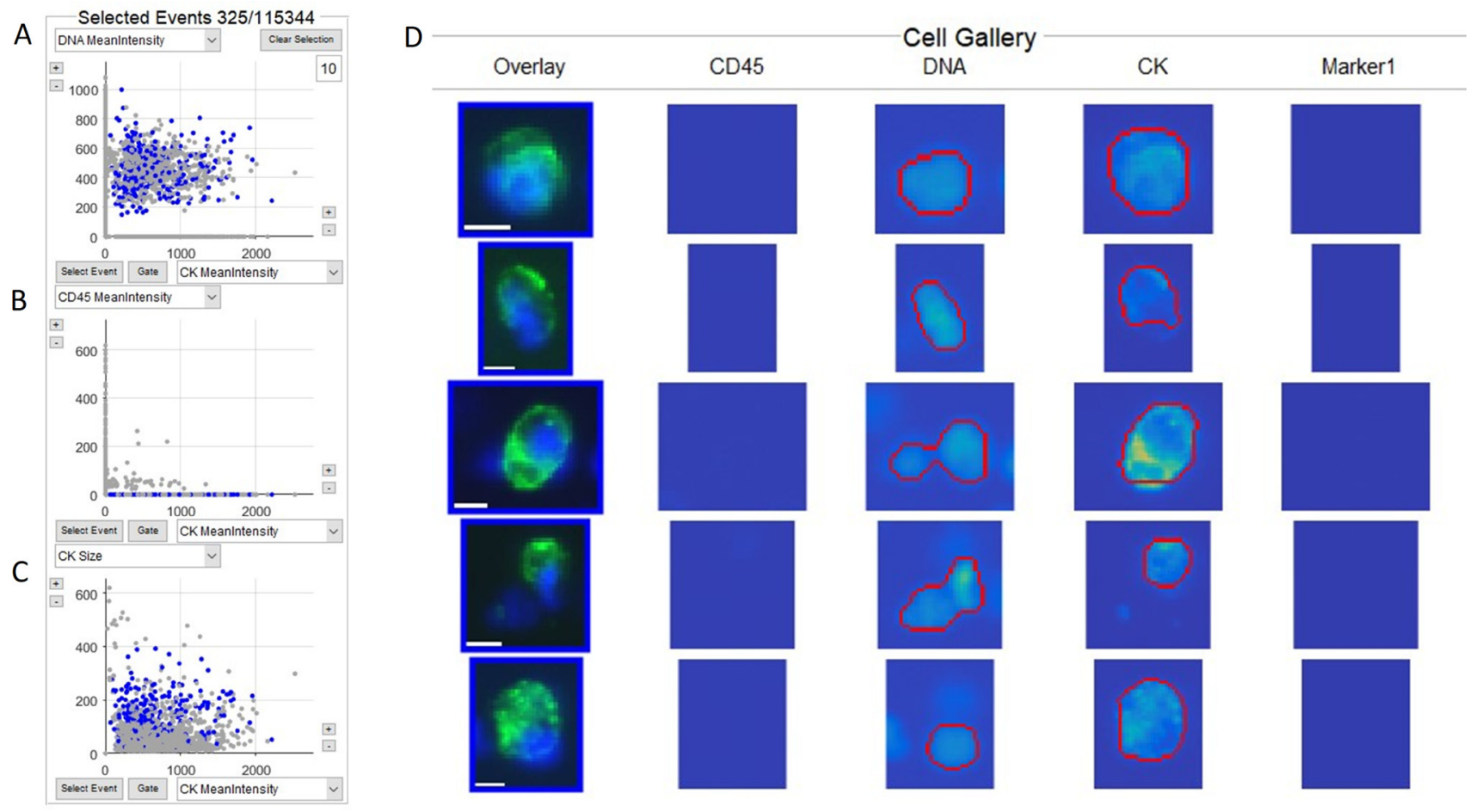

Figure 1: ACCEPT display of CTCs identified in a CRPC patient. Three scatterplots (A-C), where the objects that fall within the definition of the CTC gate (Mean Intensity CD45 $\leq 5$, Mean Intensity DNA $>45$, Mean Intensity CK $>60$, Mean Intensity Marker 1 $\leq 5$, Mean Intensity Marker $2 \leq 5,16 \leq$ Size CK $\leq 400$, DNA overlay CK $>0.2$ ), are shown as blue dots and those that fall outside of the gate are shown as grey dots. The total number of objects $(115,344)$ identified and the number of objects within the gate (325) are shown on top of Panel A. (D) shows the thumbnail images of five objects that fall within the gate. The red lines in the thumbnail identifies the area in the image in which ACCEPT identifies contours of the object in each channel. Thumbnails that do not show red contours indicate that no object in this channel could be detected. In the overlay thumbnails, DNA is represented in blue, cytokeratin (CK) in green and CD45 in red. As no CD45 is detected in these images, no red is shown in the overlay. Scale bar indicates $6.4 \mu \mathrm{m}$. 
in favorable and unfavorable groups very similar between the two data sets. For the retrospective data set, the median OS of patients with unfavorable CK18 was 11.9 months, compared to 24.2 months in the favorable group ( $p=0.001)$ with a HR of 2.4. Similarly, for the prospective data set, the patients in the unfavorable CK18 group had significantly lower survival (8.1 versus 15.4 months in the favorable group, $p=0.001$ ) with a HR of 3.5. For ccCK18 no significant difference $(p=0.48)$ in OS could be observed between the two groups regardless the cutoff value used. The summarized HRs of all variables are summarized in Supplementary Table 4.

\section{Multivariable Cox proportional hazards regression analyses}

Table 1 shows the final multivariable Cox model selected which included variables CTCs, tdEVs, CK18, prostate specific antigen (PSA), alkaline phosphatase (ALP), lactate dehydrogenase (LDH), albumin (Alb), hemoglobin $(\mathrm{Hb})$ and age. Of the known prognostic variables, LDH, albumin and hemoglobin were all selected in the final model. There was no evidence that either CK18 or ccCK18 were predictive of overall survival after LDH was included in the multivariable model. CTCs were highly correlated with tdEV (Pearson's rho $=0.79$ for the transformed variables) and were not included in the final multivariable model after tdEV was the first variable selected. At 24 months, Uno's C-Index was significantly higher in the multivariable model with tdEV $(\mathrm{C}$-Index $=0.77)$ compared to the multivariable model which included LDH, albumin and hemoglobin (C-Index $=$ 0.73 ; Difference $=0.04 ; 95 \% \mathrm{CI}=0.01$ to $0.06 ; p=0.006$ ) suggested that tdEV improved prognostic prediction. There was no evidence of a difference in the prognostic abilities of the model which included CTCs, LDH, albumin and hemoglobin (C-Index $=0.77$ ) compared to the model which included tdEVs (Difference $=-0.002$; $95 \%$ CI: -0.02 to $0.01 ; p=1.00$ )

\section{DISCUSSION}

Rapid advances in drug development and treatment of cancer patients increase the necessity for new biomarkers to assess their prognosis and response to therapy accurately and in a timely fashion. In the management of CRPC patients, adequate response to therapy is challenging as the traditional Response Evaluation Criteria in Solid Tumors [11] frequently cannot be applied. The presence or absence of CTCs has emerged as a powerful biomarker to assess prognosis and therapy response [1-5]. Although the CTC numbers measured are extremely low, several ring studies have been conducted and demonstrated the robustness of the test, though a certain level of operator bias cannot be completely eliminated [12-15]. In the original studies conducted with the CellSearch system, patient groups were divided in those with less or more than 5 CTCs per $7.5 \mathrm{~mL}$ of

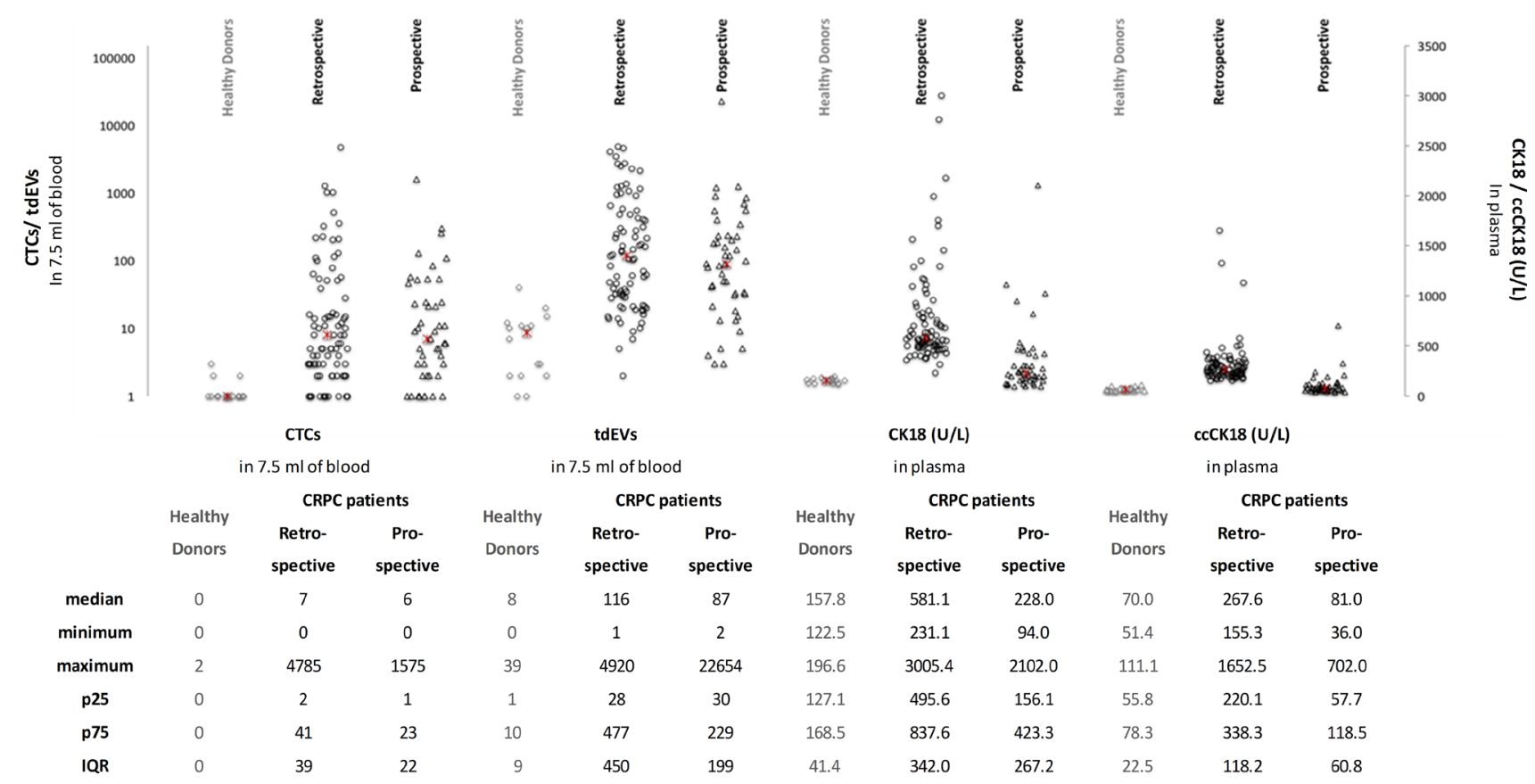

Figure 2: Number of automated CTCs and tdEVs in $7.5 \mathrm{~mL}$ of blood and plasma levels of CK18 and ccCK18 in $16 \mathrm{HDs}$, a retrospective data set of 84 CRPC patients and a prospective data set of 45 CRPC patients. The median, minimum, maximum, 25 and 75 percentiles (p25, p75 respectively) and interquartile range (IQR) values of CTCs, tdEVs, CK18 and ccCK18 are shown below the scatter plot. The median value of each variable is indicated as red $\times$ in the scatter plot. 
A

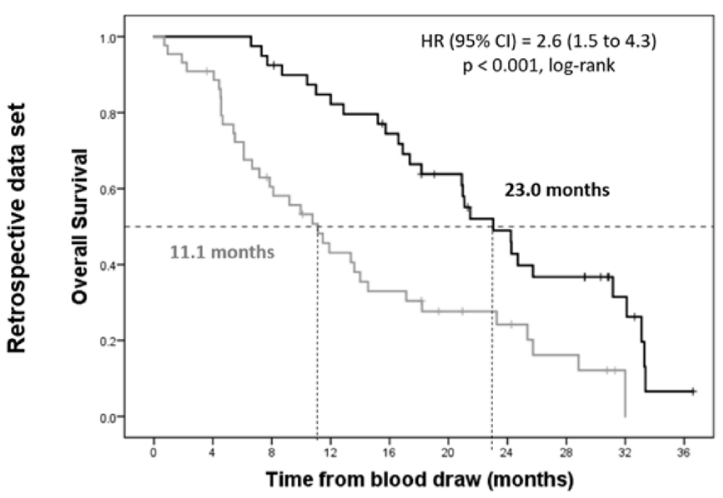

\begin{tabular}{ccccccccccc}
$<5$ CTCs & 40 & 40 & 37 & 32 & $\mathbf{2 8}$ & $\mathbf{2 2}$ & $\mathbf{1 6}$ & $\mathbf{1 2}$ & $\mathbf{6}$ & $\mathbf{1}$ \\
$\geq 5 \mathrm{CTCS}$ & 44 & 39 & 25 & 17 & 13 & 9 & 7 & 4 & 0 & 0 \\
& \multicolumn{8}{c}{ \# of patients still at risk }
\end{tabular}

B

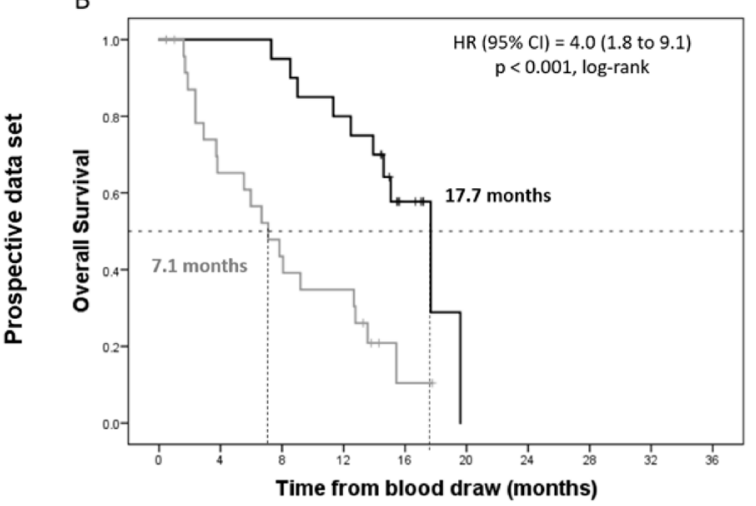

\begin{tabular}{ccccccccccc}
$<5$ CTCs & 20 & 20 & 19 & 16 & 6 & 0 & 0 & 0 & 0 & 0 \\
$\geq 5$ CTCs & 25 & 15 & 10 & 8 & 1 & 0 & 0 & 0 & 0 & 0 \\
& \multicolumn{8}{c}{ \# of patients still at risk }
\end{tabular}

C

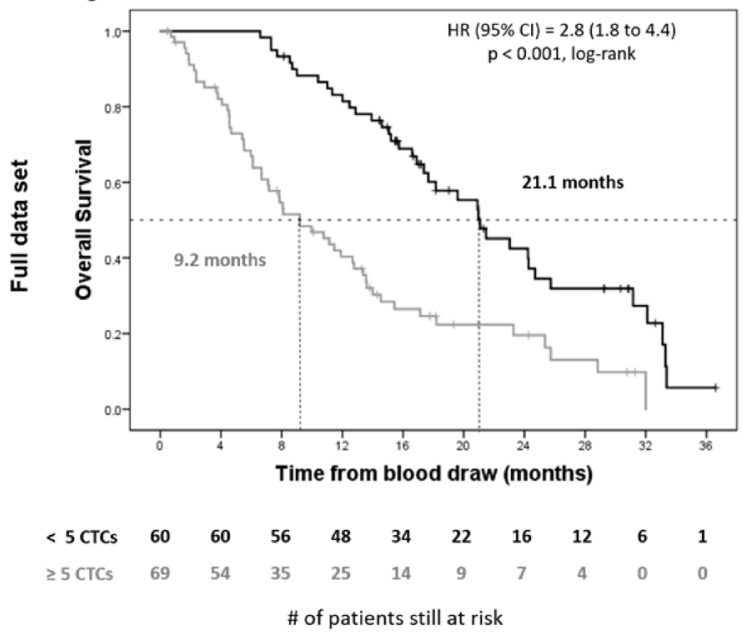

D

tdEVs

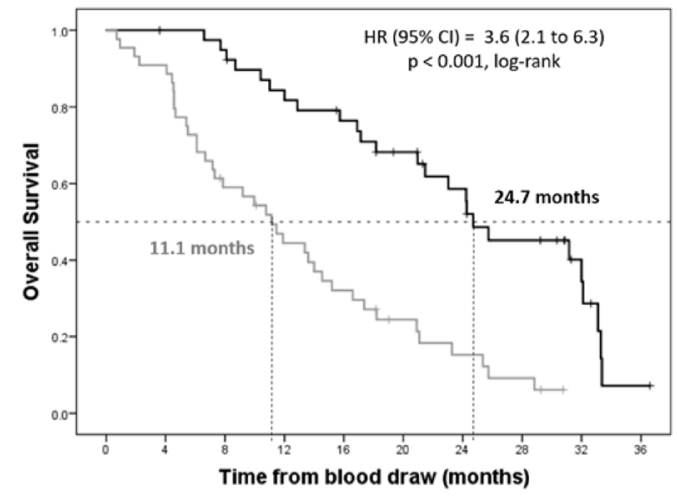

$\begin{array}{lllllllllll}<105 \text { tdEVs } & 40 & 39 & 37 & 31 & 28 & 23 & 18 & 13 & 6 & 1\end{array}$

$\begin{array}{lllllllllll}\geq 105 \text { tdEVs } & 44 & 40 & 25 & 18 & 13 & 8 & 5 & 3 & 0 & 0\end{array}$

E

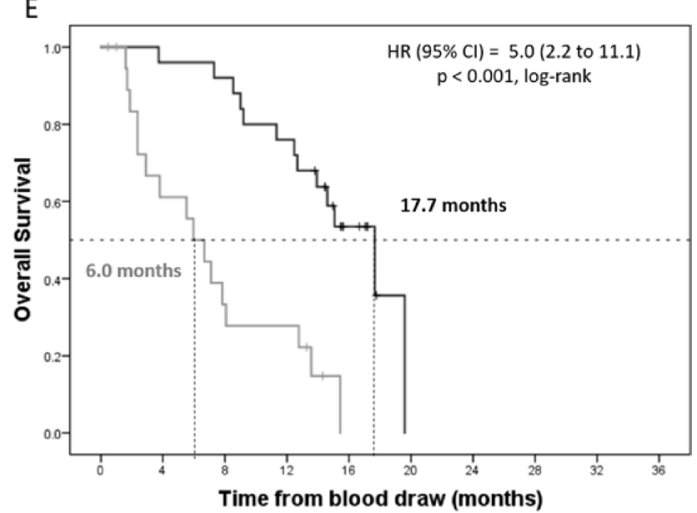

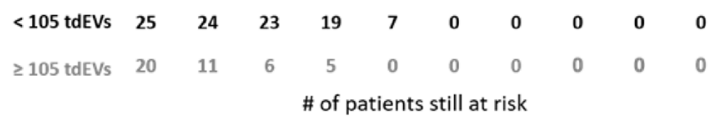

F

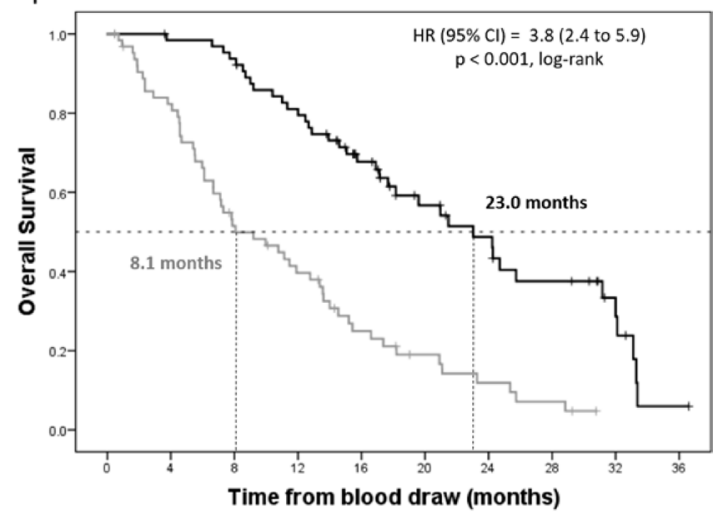

$\begin{array}{lllllllllll}<105 \text { tdEVs } & 65 & 63 & 60 & 50 & 35 & 23 & 18 & 13 & 6 & 1\end{array}$

$\begin{array}{lllllllllll}\geq 105 \text { tdEVs } & 64 & 51 & 31 & 23 & 13 & 8 & 5 & 3 & 0 & 0\end{array}$

\# of patients still at risk

Figure 3: Kaplan-Meier plots of overall survival of retrospective, prospective and full data set of CRPC patients for automated CTCs and tdEVs. Kaplan-Meier plots of overall survival of CRPC patients before initiation of therapy for automated CTCs (Panels A-C) and tdEVs (Panels D-F). The retrospective $(n=84)$, prospective $(n=45)$, and full $(n=129)$ data sets are shown in panels $\mathrm{A} / \mathrm{D}, \mathrm{B} / \mathrm{E}$, and $\mathrm{C} / \mathrm{F}$ respectively. Patients were dichotomized into unfavorable (grey lines) and favorable groups (black lines) on a cut-off value of 5 for CTCs and 105 for tdEVs in $7.5 \mathrm{~mL}$ of blood. Vertical tick marks indicate censored patients. The number of patients at risk in each group is shown under the horizontal axis. The median overall survival for each group, the Cox hazard ratio (HR), and significance $(\log$-rank $\mathrm{p})$ are indicated in each panel. 
A

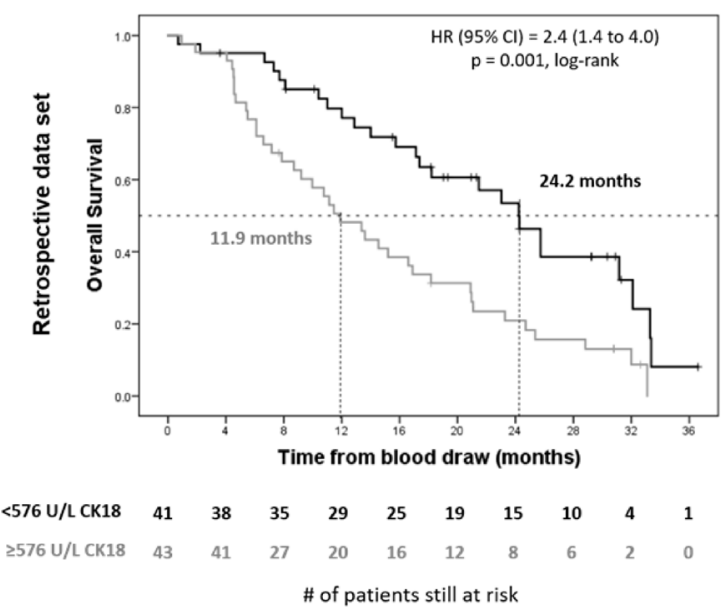

B

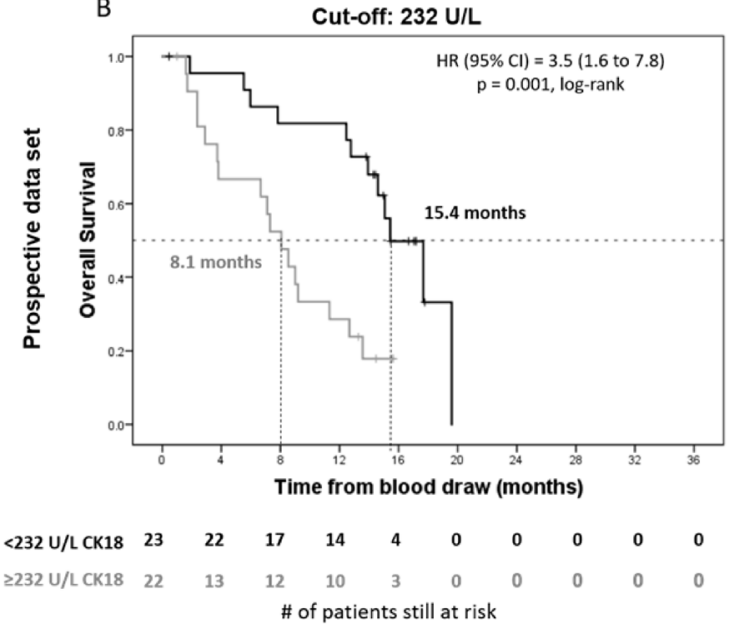

C
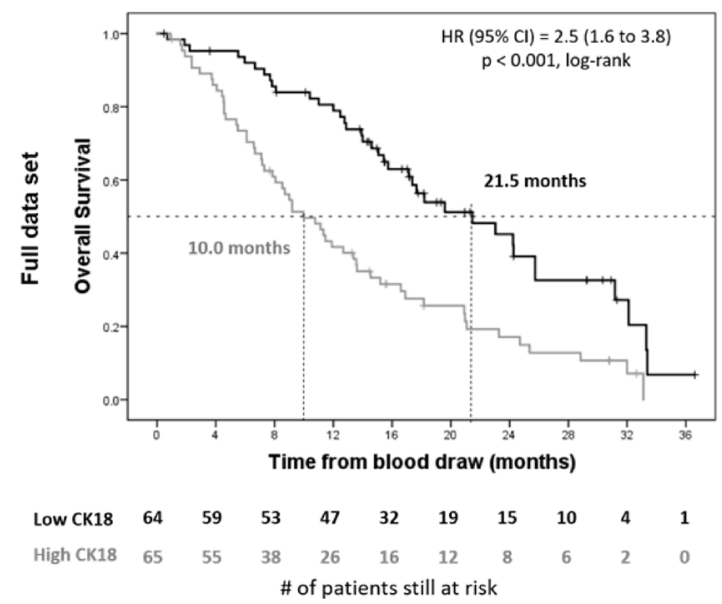

D $\quad$ ccCK18

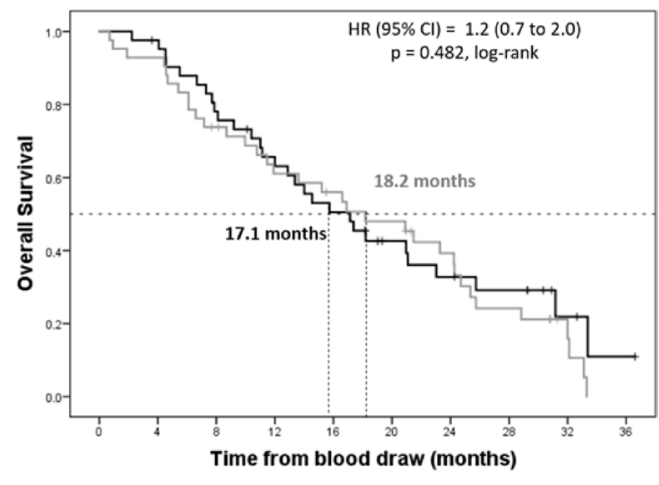

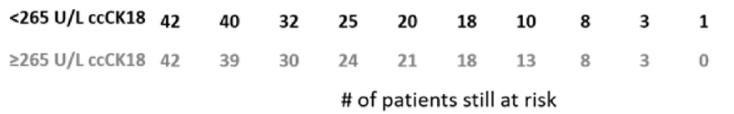

E Cut-off: 81 U/L

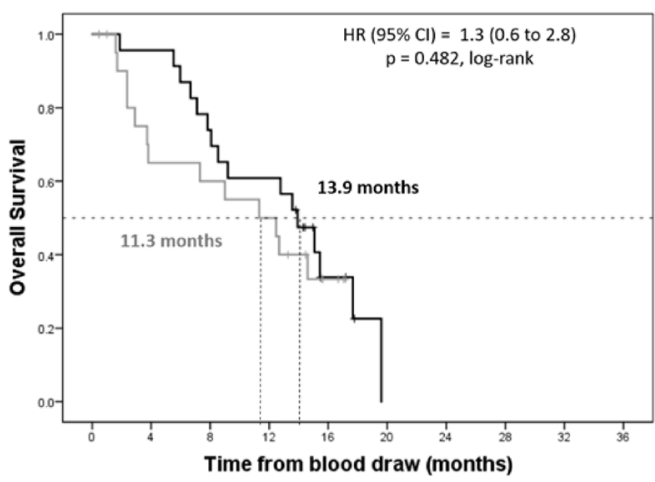

$\begin{array}{lllllllllll}<81 \text { U/L ccCK18 } & 23 & 22 & 17 & 14 & 4 & 0 & 0 & 0 & 0 & 0\end{array}$

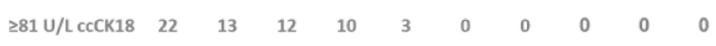

$\#$ of patients still at risk

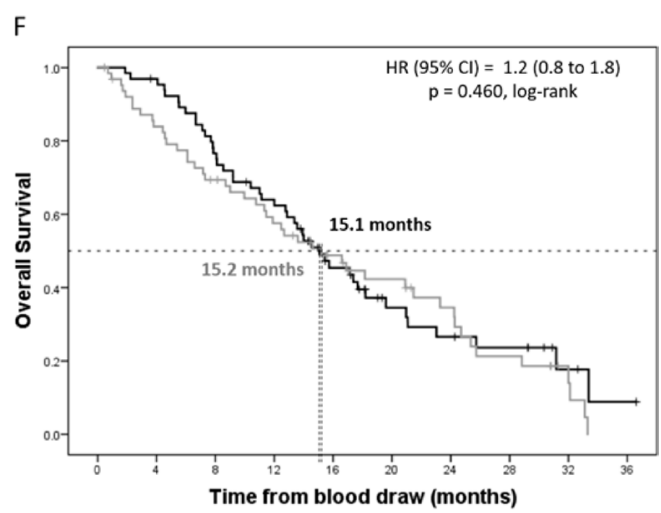

$\begin{array}{lllllllllll}\text { Low ccCK18 } & 65 & 62 & 49 & 39 & 25 & 13 & 10 & 8 & 3 & 1\end{array}$

Figure 4: Kaplan-Meier plots of overall survival of retrospective, prospective and full data set of CRPC patients for plasma levels of CK18 and ccCK18. Kaplan-Meier plots of overall survival of CRPC patients before initiation of therapy for total plasma cytokeratin 18 (CK18, Panels A-C) and caspase-cleaved plasma cytokeratin 18 (ccCK18, Panels D-F) in retrospective $(n=84$, Panels A, D), prospective ( $n=45$, Panels B, E) and full $(n=129$, Panels C, F) data sets. Patients were dichotomized into unfavorable (grey lines) and favorable groups (black lines) on a cut-off value of $576 \mathrm{U} / \mathrm{L}$ CK18 and $265 \mathrm{U} / \mathrm{L}$ ccCK18 for panels A, D. Patients were dichotomized on a cut-off value of $232 \mathrm{U} / \mathrm{L}$ CK1 18 and $81 \mathrm{U} / \mathrm{L}$ ccCK1 8 for panels (B, E). Dichotomization on the full data set (Panels C, F) was done using the different cut-off values for each data set. Vertical tick marks indicate censored patients. The number of patients at risk in each group is shown under the horizontal axis. The median overall survival for each group, the Cox hazard ratio (HR), and significance $(\log$-rank $\mathrm{p})$ are indicated in each panel. 
Table 1: Multivariable cox proportional hazards regression analysis for full data set of CRPC patients

\begin{tabular}{lcccc}
\hline Variables in equation & HR & $\mathbf{9 5 \%}$ & CI & $\boldsymbol{p}$-value \\
\hline Albumin $(\mathrm{g} / \mathrm{dl})$ & 0.38 & 0.21 & 0.67 & 0.001 \\
Hemoglobin $(\mathrm{g} / \mathrm{l})$ & 0.81 & 0.69 & 0.94 & 0.006 \\
$\operatorname{tdEV}(\ln (0.0001+\operatorname{count} / 1000))$ & 1.30 & 1.11 & 1.51 & 0.001 \\
$\mathrm{LDH}(\ln (0.001+\mathrm{U} / \mathrm{L} / 100))$ & 1.84 & 1.09 & 3.12 & 0.023 \\
\hline
\end{tabular}

( $N=118,11$ cases were dropped due to missing values).

blood and shown to have different clinical outcomes [1-5]. However, recently it was demonstrated that the actual number of CTCs -and not just a count above or below a selected cut-off value- is of importance in order to assess response to therapy $[4,5,15]$. That fact makes the need for accuracy of the actual CTC count more imperative.

Towards that direction, we previously reported the use of image analysis algorithms to eliminate the operator bias and automatically identify CTCs in the CellSearch generated images [16]. Efforts to improve these algorithms have continued and have led to the image analysis program ACCEPT. ACCEPT enables a quantitative definition of objects, such as CTCs and tdEVs, derived from the images using specific parameters. In the present study, an excellent correlation $\left(\mathrm{R}^{2}=0.88\right)$ was found (Supplementary Figure 2) between the manual (obtained by the operator) and the automated CTC counts (obtained by ACCEPT).

To define the cut-off values dichotomizing patients into groups of higher and lower risk by ACCEPT automated CTC and tdEV counts, a retrospective data set of 84 CRPC patients from the original IMMC38 study was used. ROC analysis in that data set (Supplementary Figure 3) showed a cut-off value of 5 CTCs in $7.5 \mathrm{~mL}$ of whole blood that stratified patients into favorable and unfavorable groups (Figure 3). The CTC cut-off was validated in a prospective data set of 45 newly enrolled CRPC patients. In the full data set, patients of the unfavorable group (with $\geq 5$ CTCs) had significantly shorter median OS compared to patients of the favorable group ( $p<0.001)$ with a HR of 2.8 (95\% CI: 1.8 to 4.4$)$.

In the CellSearch image analysis algorithms, objects expressing both Cytokeratin as well as DAPI are presented to the operator, whereas the majority of tdEVs is missed, as the latter ones do not have DAPI signal. Using the automated ACCEPT identification of tdEVs however, the laborintensive manual review of the original stack of 144-180 of fluorescence images for each patient sample [7] can be replaced by a process with perfect repeatability. In our study, there was a strong correlation between automated CTC and tdEV counts (Supplementary Figure 2) and their presence in higher amounts was strongly correlated with poor clinical outcome (Figure 3).

Since tdEVs were isolated with the CellSearch system, they were enriched from the blood fraction centrifuged at $800 \mathrm{~g}$ based on their EpCAM expression and were detected by the expression of Cytokeratins. However, the tdEV number in the plasma should be much higher because the vast majority of these EVs have a diameter below $4 \mu \mathrm{m}$ [17]. Moreover, the CellSearch Analyzer was not designed for the detection of small particles, and the fraction of tdEVs below the CellSearch detection limit is unknown. Further investigation is recommended for the isolation and detection of tdEVs from plasma of patients and their correlation with clinical outcome.

To assess the clinical relevance of soluble Cytokeratins in plasma, CK18 and ccCK18 were determined by M65 and M30 ELISAs respectively. Several studies have shown the significantly elevated (cc)CK18 levels in serum/plasma of different cancer patients compared to the respective values of healthy donors [24-26]. Moreover, CK18 and ccCK18 have been used in several studies to evaluate the induced cell death modes and antitumor activity of different drug treatments [8, 18-21]. Interestingly, in a previous study, both ccCK18 and CK18 levels in plasma were predictive of the clinical outcome of small cell lung cancer patients [22]. In another study on both small and non-small cell lung cancer patients, the serum levels of ccCK18 were prognostic for OS [23]. A third study demonstrated significantly increased levels of ccCK18 in the sera of breast cancer patients compared to HDs but without any association of ccCK18 with the OS of these patients [26]. In our study in CRPC patients, we found that both CK18 and ccCK18 are elevated in patients compared to HDs, but only CK18 is prognostic for OS (Figure 4). It is worth mentioning that the ELISA assays we applied for CK18 and ccCK18 do not involve a detergent and thus measure only the soluble proteins and do not detect CK18 or ccCK18 inside tdEVs. Adding a detergent to the plasma may solubilize the tdEVs, and potentially result in a prognostic ccCK18 because a portion of cytokeratins in CTCs and tdEVs is caspase-cleaved as shown by M30 expression [27].

In summary, our findings suggest that ACCEPT software allows fast enumeration of well defined objects as CTCs and tdEVs eliminating interoperator bias. The enumeration of tdEVs in CRPC patients can provide prognostic information equivalent to CTCs in CRPC disease. tdEVs were typically detected at a 20 
times higher frequencies in $7.5 \mathrm{ml}$ of blood compared to CTCs so tdEVs may offer increased utility. The association between OS and CK18 in CRPC is not as strong as CTCs and tdEVs based on the respective HRs. The multivariable analysis of the full data set of CRPC patients including CTCs, tdEVs, CK18 and ccCK18 and traditional biomarkers such as age, PSA, ALP, LDH, Alb and $\mathrm{Hb}$ resulted in a final model with $\mathrm{LDH}$, albumin, hemoglobin and tdEVs. tdEVs improved significantly the prognostic prediction of the patients. Neither CK18 nor ccCK18 were predictive of OS after LDH inclusion. CTCs were highly correlated with tdEVs so were not present in the final multivariable model but provided equivalent prognostic information. To predict whether a certain treatment will be effective, assessment of the treatment target will need to be assessed. Preferably one would examine tumor cells representing the various metastatic sites, but as that is practically not feasible, CTCs can provide this information provided that one can isolate them in sufficient quantity [28, 29]. Due to the fact that most patients have single-digit CTC counts, the higher number of tdEVs could render them to a promising surrogate biomarker for the assessment of changes of tumor load (through their rises and declines) in response to therapy over time and even in personalized therapeutics by proceeding with the downstream analysis of their protein and RNA cargo.

\section{MATERIALS AND METHODS}

\section{Patients and healthy donors}

Blood from 16 anonymous HDs was obtained after written informed consent. These samples were used to determine the baseline values of CTCs, tdEVs, CK18 and ccCK18.

All patients had histologically confirmed metastatic prostate cancer progressing despite castrate levels of testosterone and had provided written informed consent to trial protocols approved by the institutional review boards at each participating center. Two patient cohorts enrolled in different studies and during different time periods were included in the present study. More specifically, the retrospective data set consisted of 84 CRPC patients who were starting a new line of therapy and were enrolled in the IMMC-38 study [1] out of the 231 evaluable patients of IMMC38 study, because stored EDTA plasma of only these patients was available for further (cc) CK18 assessment. The digitally stored CellSearch images were also available.

The prospective data set consisted of 45 CRPC patients who were starting a new line of therapy at the Royal Marsden Hospital, and from whom stored plasma samples in CellSave and digitally stored CellSearch images were available.

\section{Sample collection and preparation}

For the retrospective data set, $7.5 \mathrm{~mL}$ of blood was collected in CellSave blood collection tubes (Menarini, Huntingdon Valley, PA, USA) and 5-10 $\mathrm{mL}$ of blood in in EDTA tubes. For CTC and tdEV assessment, CellSave blood was processed with the CellSearch Autoprep within 96 hours from the time of blood draw. For CK18 and ccCK18 assessment, EDTA blood was centrifuged for 10 minutes at $1710 \mathrm{~g}$ without brake within 24 hours from the time of blood draw and plasma was collected and stored at $-80^{\circ} \mathrm{C}$ until further use. The plasma samples of the retrospective data set were assessed for CK18 and ccCK18 within 5 years from their collection.

For both the prospective data set as well as the healthy donors, $7.5 \mathrm{~mL}$ of blood was collected in CellSave tubes, and was processed within 96 hours after collection. More specifically, the blood samples were centrifuged for 10 minutes at $800 \mathrm{~g}$ without brake and $0.5-2.0 \mathrm{~mL}$ of plasma was collected without disturbing the buffy coat, and stored at $-80^{\circ} \mathrm{C}$ directly after collection until further use. The remaining blood sample was processed with the CellSearch system. The plasma samples of the HDs and the prospective data set were assessed for CK18 and ccCK18 levels within 1 year from their collection.

\section{Isolation and detection of CTCs and tdEVs}

The CellSearch system (Menarini, Huntingdon Valley, PA, USA) was used to isolate and detect CTCs and tdEVs. The system consists of the CellTracks Autoprep ${ }^{\circledR}$ and the CellTracks Analyzer II ${ }^{\circledR}$. The CellTracks Autoprep ${ }^{\circledR}$ immunomagnetically enriches EpCAM+ objects from blood and stains the enriched objects with the nuclear dye DAPI, phycoerythrin conjugated antibodies against cytokeratin 8, 18 and 19 (CK-PE) and allophycocyanin conjugated antibody against the leukocyte specific marker CD45 (CD45-APC). The enriched labeled objects are contained in a cartridge, which is placed in a CellTracks Magnest. The CellTracks Analyzer $\mathrm{II}^{\circledR}$ captures digital images with four different fluorescent channels using a $10 \times / 0.45 \mathrm{NA}$ objective and a charge-coupled device camera with $6.7 \times$ $6.7 \mu \mathrm{m}$ sized pixels. For each cartridge, 144-180 4-layer tiff images of DAPI, FITC, CK-PE, CD45-APC are saved.

\section{Enumeration of CTCs and tdEVs}

The CTCs and tdEVs, were enumerated using the open-source ACCEPT software (http://github.com/ LeonieZ/ACCEPT) developed in the frames of CTCTrap (www.utwente.nl/en/tnw/ctctrap/) and CANCER-ID EU (www.cancer-id.eu) programs. Briefly, the digitally stored CellSearch fluorescence images are processed by ACCEPT to identify objects using multiscale segmentation [10]. Objects can be categorized using configurable criteria, and objects within a category are 
shown in a gallery of images as well as in scatter plots using the various parameters measured from the objects. An example of the scatterplots and images of CTCs is shown in Figure 1. The scatterplots and images of tdEVs are shown in Supplementary Figure 1. Both gates used for CTC and tdEV enumeration are mentioned in the legends of Figure 1 and Supplementary Figure 1 respectively.

\section{Measurement of CK18 and ccCK18 concentrations}

CK18 present in epithelial cells is cleaved by caspases during apoptosis. Thus, the amount of caspase cleaved CK18 is related to apoptosis, while the total amount of CK18 is related to the sum of CK18 due to apoptosis, necrosis and present in viable cells. Two commercially available ELISA kits, namely M65 and M30-Apoptosense (VLVBio, Nacka, Sweden) were used to measure the levels of soluble ccCK18, and CK18 respectively in plasma samples of healthy donors and CRPC patients. The aforementioned ELISA assays have been already used for clinical assays [18, 19, 22, 24, 25]. The M65 assay uses two different monoclonal antibodies to recognize intact and caspase-cleaved CK18. The M30 assay uses a monoclonal antibody to recognize the neoepitope M30, which is exposed after caspase-cleavage of CK18. Both assays have a 96-well plate format and include 7 standards of defined antigen concentrations and both a low and a high concentration quality control (QC). The assays were performed according to manufacturer's instructions. Briefly, technical duplicates of $25 \mu \mathrm{l}$ were added to wells coated with a mouse monoclonal capture antibody. Next, $75 \mu$ of Horse Radish Peroxidase HRPconjugated detection antibody solution was added. After a 2 hour incubation at room temperature (RT) with constant shaking, five sequential washing steps were performed to remove unbound antibody. Next, 20 minutes incubation

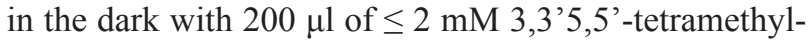
benzidine (TMB) solution resulted in color development proportional to the antigen concentration. The reaction was stopped by the addition of $50 \mu \mathrm{l}$ of $1 \mathrm{M}$ sulfuric acid and the $450 \mathrm{~nm}$ absorbance was measured within 5-30 minutes using a microplate reader. All values were corrected for the blank (background) absorbance. After calibration with the standards of defined antigen concentrations, the average of the technical duplicates was converted to concentration in $\mathrm{U} / \mathrm{L}$.

\section{Statistical analysis}

Statistical analysis was performed in SPSS 23.0 (SPSS Inc., Chicago, IL, USA) and Stata v15.1 (StataCorp, College Station, Texas, USA). To determine whether CTCs, tdEVs, CK18 and ccCK18 can be related to each other through a monotonic function, we performed a two-tailed Spearman's Rho test. To assess if there were significant differences between groups (healthy donors vs CRPC patients and retrospective vs prospective data sets) in the examined continuous variables (namely CTCs, tdEVs, CK18 and ccCK18), we performed the nonparametric Mann-Whitney $U$ test. To determine the cut-off values to dichotomize the retrospective data set into favorable and unfavorable groups we used Receiver Operating Characteristic (ROC) curves treating survival time as the reference value dichotomized by the median survival time. We set dichotomization cut-off values for CTCs, tdEVs, CK18, and ccCK18 on the value that led to equal sensitivity and specificity (minimum |sensitivity-specificity|). Overall Survival (OS) was defined as the elapsed time in months between blood draw and death. The patients who were lost to follow-up were censored. Median OS was determined by Kaplan-Meier survival curves, and Kaplan-Meier survival curves were compared using the log-rank test. Cox regression models, with each dataset included as a shared frailty parameter, were used to determine univariable and multivariable hazards ratios (HR) for OS with $95 \%$ confidence intervals (CI) for each dichotomized variable.

A final multivariable Cox model with each dataset included as a shared frailty parameter was fit including all characteristics as continuous variables. CK 18 and ccCK 18 were included with an interaction term for each dataset to account for the different methods in plasma collection. CTC, tdEV, ALP and LDH were log transformed to achieve better model fit. Due to correlation between variables the final model was selected using forward stepwise elimination ( $p_{\text {in }}=0.05$ and $p_{\text {out }}=0.10$ ). The value of tdEV in the model was assessed by calculating Uno et al. C-Index [30]. Bootstrapping $(n=1000)$ was used to calculate the $95 \%$ confidence interval and the difference, delta, between c-indeces of each of the models.

\section{Abbreviations}

ALP: ALkaline Phosphatase; APC: AlloPhycoCyanin; ccCK18: caspase-cleaved CytoKeratin 18; CI: Confidence Interval; CK: CytoKeratin; CK18: CytoKeratin 18; CD45: Cluster of Differentiation 45; CRPC: Castration-Resistant Prostate Cancer; CTC: Circulating Tumor Cell; DAPI: 4',6-DiAmidino-2-PhenylIndole; DNA: DeoxyriboNucleic Acid; ECOG ps: Eastern Cooperative Oncology Group performance status ECTM: Experimental Centre for Technical Medicine; EDTA: EthyleneDiamineTetraacetic Acid; ELISA: Enzyme-Linked ImmunoSorbent Assay; EpCAM: Epithelial Cell Adhesion Molecule; FITC: Fluorescein IsoThioCyanate; HD: Healthy Donor; HR: Hazard Ratio; HRP: Horse Radish Peroxidase; IQR: InterQuartile Range; LDH: Lactate DeHydrogenase; OS: Overall Survival; PE: PhycoErythrin; PSA: Prostate Specific Antigen; QC: Quality Control; ROC: Receiver Operating Characteristic; RT: Room Temperature; tdEV: tumor-derived Extracellular Vesicle; TMB: 3,3'5,5'-TetraMethyl-Benzidine 


\section{Author contributions}

Mariane Souse Fontes, Pasquale Rescigno and Wendy Onstenk helped with clinical data collection; Gemma Fowler and Penny Flohr helped with CTC isolation, plasma collection and sample shipment. Statistical analysis was performed by David Dolling, Afroditi Nanou, Frank AW Coumans and Guus van Dalum. ACCEPT image analysis program was developed by Leonie L Zeune, Guus van Dalum and Christoph Brune. CTC analysis was performed by Mateus Crespo, Wendy Onstenk and Afroditi Nanou. Plasma CK assays were performed by Wendy Onstenk and Afroditi Nanou. Study was designed and manuscript was written by Stefan Sleijfer, Johann S de Bono, Afroditi Nanou, Frank AW Coumans, Guus van Dalum and Leon WMM Terstappen.

\section{ACKNOWLEDGMENTS}

The authors acknowledge the Developmental BioEngineering department of the University of Twente for providing access to their microplate absorbance reader and the Experimental Centre for Technical Medicine (ECTM) of the University of Twente for providing the healthy donor samples.

\section{CONFLICTS OF INTEREST}

The authors declare no potential conflicts of interest.

\section{FUNDING}

Afroditi Nanou is supported by NWO Applied and Engineering Sciences project Cancer-ID \#14197, Leonie L. Zeune is supported by the EUFP7 project \#305341 CTCTrap and the EU IMI project \# 115749-1 CANCERID. Mateus Crespo was supported by EUFP7 project \#305341 CTCTrap.

\section{REFERENCES}

1. de Bono JS, Scher HI, Montgomery RB, Parker C, Miller MC, Tissing H, Doyle GV, Terstappen LW, Pienta KJ, Raghavan D. Circulating tumor cells predict survival benefit from treatment in metastatic castration-resistant prostate cancer. Clin Cancer Res. 2008; 14:6302-09. https:// doi.org/10.1158/1078-0432.CCR-08-0872.

2. Scher HI, Heller G, Molina A, Attard G, Danila DC, Jia X, Peng W, Sandhu SK, Olmos D, Riisnaes R, McCormack R, Burzykowski T, Kheoh $\mathrm{T}$, et al. Circulating tumor cell biomarker panel as an individual-level surrogate for survival in metastatic castration-resistant prostate cancer. J Clin Oncol. 2015; 33:1348-55. https://doi.org/10.1200/ JCO.2014.55.3487.
3. Goldkorn A, Ely B, Quinn DI, Tangen CM, Fink LM, $\mathrm{Xu}$ T, Twardowski P, Van Veldhuizen PJ, Agarwal N, Carducci MA, Monk JP 3rd, Datar RH, Garzotto M, et al. Circulating tumor cell counts are prognostic of overall survival in SWOG S0421: a phase III trial of docetaxel with or without atrasentan for metastatic castrationresistant prostate cancer. J Clin Oncol. 2014; 32:1136-42. https://doi. org/10.1200/JCO.2013.51.7417.

4. Lorente D, Olmos D, Mateo J, Bianchini D, Seed G, Fleisher M, Danila DC, Flohr P, Crespo M, Figueiredo I, Miranda S, Baeten K, Molina A, et al. Decline in circulating tumor cell count and treatment outcome in advanced prostate cancer. Eur Urol. 2016; 70:985-92. https://doi. org/10.1016/j.eururo.2016.05.023.

5. Lorente D, Ravi P, Mehra N, Pezaro C, Omlin A, Gilman A, Miranda M, Rescigno P, Kolinsky M, Porta N, Bianchini D, Tunariu N, Perez R, et al. Interrogating metastatic prostate cancer treatment switch decisions: a multi-institutional survey. Eur Urol Focus. 2016 Oct 10. [Epub ahead of print]. https://doi.org/10.1016/j.euf.2016.09.005.

6. Miyamoto DT, Sequist LV, Lee RJ. Circulating tumour cells-monitoring treatment response in prostate cancer. Nat Rev Clin Oncol. 2014; 11:401-12. https://doi. org/10.1038nrclinonc.2014.82 https://doi.org/10.1038/ nrclinonc.2014.82.

7. Coumans FA, Doggen CJ, Attard G, de Bono JS, Terstappen LW. All circulating EpCAM+CK + CD45-objects predict overall survival in castration-resistant prostate cancer. Ann Oncol. 2010; 21:1851-57. https://doi.org/10.1093/annonc/ mdq030.

8. Kramer G, Erdal H, Mertens HJ, Nap M, Mauermann J, Steiner G, Marberger M, Bivén K, Shoshan MC, Linder S. Differentiation between cell death modes using measurements of different soluble forms of extracellular cytokeratin 18. Cancer Res. 2004; 64:1751-56. https://doi. org/10.1158/0008-5472.CAN-03-2455.

9. Allard WJ, Matera J, Miller MC, Repollet M, Connelly MC, Rao C, Tibbe AG, Uhr JW, Terstappen LW. Tumor cells circulate in the peripheral blood of all major carcinomas but not in healthy subjects or patients with nonmalignant diseases. Clin Cancer Res. 2004; 10:6897-904.https://doi. org/10.1158/1078-0432.CCR-04-0378.

10. Zeune L, van Dalum G, Terstappen L, van Gils S, Brune C. Multiscale segmentation via Bregman distances and nonlinear spectral analysis. SIAM J Imaging Sci. 2017; 10:111-46. https://doi.org/10.1137/16M1074503.

11. Eisenhauer EA, Therasse P, Bogaerts J, Schwartz LH, Sargent D, Ford R, Dancey J, Arbuck S, Gwyther S, Mooney M, Rubinstein L, Shankar L, Dodd L, et al. New response evaluation criteria in solid tumours: revised RECIST guideline (version 1.1). Eur J Cancer. 2009; 45:228-47. https://doi.org/10.1016/j.ejca.2008.10.026.

12. Ignatiadis $\mathrm{M}$, Riethdorf $\mathrm{S}$, Bidard $\mathrm{FC}$, Vaucher I, Khazour M, Rothé F, Metallo J, Rouas G, Payne RE, 
Coombes R, Teufel I, Andergassen U, Apostolaki S, et al. International study on inter-reader variability for circulating tumor cells in breast cancer. Breast Cancer Res. 2014; 16:R43. https://doi.org/10.1186/bcr3647.

13. Riethdorf S, Fritsche H, Müller V, Rau T, Schindlbeck C, Rack B, Janni W, Coith C, Beck K, Jänicke F, Jackson S, Gornet T, Cristofanilli M, Pantel K. Detection of circulating tumor cells in peripheral blood of patients with metastatic breast cancer: a validation study of the CellSearch system. Clin Cancer Res. 2007; 13:920-28. https://doi. org/10.1158/1078-0432.CCR-06-1695.

14. Kraan J, Sleijfer S, Strijbos MH, Ignatiadis M, Peeters D, Pierga JY, Farace F, Riethdorf S, Fehm T, Zorzino L, Tibbe AG, Maestro M, Gisbert-Criado R, et al. External quality assurance of circulating tumor cell enumeration using the CellSearch $\left({ }^{\circledR}\right)$ system: a feasibility study. Cytometry B Clin Cytom. 2011; 80:112-18. https://doi.org/10.1002/ cyto.b.20573.

15. Coumans FA, Ligthart ST, Terstappen LW. Interpretation of changes in circulating tumor cell counts. Transl Oncol. 2012; 5:486-91. https://doi.org/10.1593/tlo.12247.

16. Ligthart ST, Coumans FA, Attard G, Cassidy AM, de Bono JS, Terstappen LW. Unbiased and automated identification of a circulating tumour cell definition that associates with overall survival. PLoS One. 2011; 6:e27419. https://doi.org/10.1371/journal.pone.0027419.

17. van der Pol E, Coumans FA, Grootemaat AE, Gardiner C, Sargent IL, Harrison P, Sturk A, van Leeuwen TG, Nieuwland R. Particle size distribution of exosomes and microvesicles determined by transmission electron microscopy, flow cytometry, nanoparticle tracking analysis, and resistive pulse sensing. J Thromb Haemost. 2014; 12:1182-92. https://doi.org/10.1111/jth.12602.

18. de Haas EC, di Pietro A, Simpson KL, Meijer C, Suurmeijer AJ, Lancashire LJ, Cummings J, de Jong S, de Vries EG, Dive C, Gietema JA. Clinical evaluation of M30 and M65 ELISA cell death assays as circulating biomarkers in a drugsensitive tumor, testicular cancer. Neoplasia. 2008; 10:1041-48. https://doi.org/10.1593/neo.08620.

19. Cummings J, Hodgkinson C, Odedra R, Sini P, Heaton SP, Mundt KE, Ward TH, Wilkinson RW, Growcott J, Hughes A, Dive C. Preclinical evaluation of M30 and M65 ELISAs as biomarkers of drug induced tumor cell death and antitumor activity. Mol Cancer Ther. 2008; 7:455-63. https://doi.org/10.1158/1535-7163.MCT-07-2136.

20. Pichon MF, Labroquère M, Rezaï K, Lokiec F. Variations of soluble fas and cytokeratin 18-Asp 396 neo-epitope in different cancers during chemotherapy. Anticancer Res. 2006; 26:2387-92.
21. Kramer G, Schwarz S, Hagg M, Havelka A, Linder S. Docetaxel induces apoptosis in hormone refractory prostate carcinomas during multiple treatment cycles. Br J Cancer. 2006; 94:1592-98. https://doi.org/10.1038/sj.bjc.6603129.

22. Hou JM, Greystoke A, Lancashire L, Cummings J, Ward T, Board R, Amir E, Hughes S, Krebs M, Hughes A, Ranson M, Lorigan P, Dive C, Blackhall FH. Evaluation of circulating tumor cells and serological cell death biomarkers in small cell lung cancer patients undergoing chemotherapy. Am J Pathol. 2009; 175:808-16. https://doi.org/10.2353/ ajpath.2009.090078.

23. Ulukaya E, Yilmaztepe A, Akgoz S, Linder S, Karadag M. The levels of caspase-cleaved cytokeratin 18 are elevated in serum from patients with lung cancer and helpful to predict the survival. Lung Cancer. 2007; 56:399-404. https://doi. org/10.1016/j.lungcan.2007.01.015.

24. Tas F, Karabulut S, Yildiz I, Duranyildiz D. Clinical significance of serum M30 and M65 levels in patients with breast cancer. Biomed Pharmacother. 2014; 68:1135-40. https://doi.org/10.1016/j.biopha.2014.10.021.

25. Ozturk B, Coskun U, Sancak B, Yaman E, Buyukberber S, Benekli M. Elevated serum levels of M30 and M65 in patients with locally advanced head and neck tumors. Int Immunopharmacol. 2009; 9:645-48. https://doi. org/10.1016/j.intimp.2009.02.004.

26. Ueno T, Toi M, Bivén K, Bando H, Ogawa T, Linder S. Measurement of an apoptotic product in the sera of breast cancer patients. Eur J Cancer. 2003; 39:769-74. https://doi. org/10.1016/S0959-8049(02)00865-1.

27. Larson CJ, Moreno JG, Pienta KJ, Gross S, Repollet M, O'Hara SM, Russell T, Terstappen LW. Apoptosis of circulating tumor cells in prostate cancer patients. Cytometry A. 2004; 62:46-53. https://doi.org/10.1002/ cyto.a.20073.

28. Coumans FA, Ligthart ST, Uhr JW, Terstappen LW. Challenges in the enumeration and phenotyping of CTC. Clin Cancer Res. 2012; 18:5711-18. https://doi. org/10.1158/1078-0432.CCR-12-1585.

29. Stoecklein NH, Fischer JC, Niederacher D, Terstappen LW. Challenges for CTC-based liquid biopsies: low CTC frequency and diagnostic leukapheresis as a potential solution. Expert Rev Mol Diagn. 2016; 16:147-64. https:// doi.org/10.1586/14737159.2016.1123095.

30. Uno H, Cai T, Pencina M, D’Agostino R, Wei L. On the Cstatistics for evaluating overall adequacy of risk prediction procedures with censored survival data. Stat Med. 2011; 30:1105-17. https://doi.org/10.1002/sim.4154. 